\title{
OPINIÓN
}

\section{TERRORISMO COMO DETONANTE DE DESASTRES: ATENTADOS TERRORISTAS CONTRA OLEODUCTO CAÑO LIMÓN COVEÑAS EN COLOMBIA}

\author{
Javier Augusto Vera Solano ${ }^{1^{*}}$
}

\section{INTRODUCCIÓN}

El negocio de hidrocarburos en Colombia es complejo, son muchos las amenazas a los que se encuentra expuesto; riesgos internos, externos, naturales, económicos, entre otros; y todos estos generan unos desafíos que deben ser atendidos de manera específica para ser estudiados, entenderlos y generar mecanismos de mitigación respectivamente. Si miramos los aspectos de seguridad, el riesgo que mayor impacto genera en Colombia son los atentados terroristas (Issa Tejada, 2015).

Desde un punto de vista particular, un desastre es el daño grave o la alteración considerable de las condiciones normales de vida en un área geográfica determinada, gatillado por fenómenos naturales y por efectos catastróficos de la acción humana, que requiere por ello de la especial atención de los organismos del Estado y de otras entidades de carácter humanitario 0 de servicio social. Entonces los atentados terroristas a la infraestructura petrolera en este caso, el oleoducto Caño Limón Coveñas, podrían entenderse como 'desastres ambientales'. Así mismo, es importante señalar que los desastres también pueden tener un origen humano como las guerras y los conflictos civiles que afectan severamente a comunidades y desplazan a la gente de sus hogares (Gobernación del Casanare, 2011). Desde esta perspectiva, el conflicto que actualmente se presenta en Colombia con los grupos armados al margen de la ley se puede incluir dentro de las detonantes de un desastre con impactos severos económicos, políticos, sociales y también ambientales.

Cifras de Ecopetrol (Empresa Colombiana de Petróleos), evidencian que, durante los últimos 40 años, aproximadamente, la infraestructura petrolera del país ha sido atacada en cerca de 2800 oportunidades, lo cual ha generado el derrame de más de 3,7 millones de barriles de crudo. Los atentados al Oleoducto Caño Limón-Coveñas, según las cifras de Ecopetrol, han afectado más de 65.000 metros cuadrados de suelo y cerca de 40.500 metros lineales de cuerpos de agua. Los mayores impactos se han registrado en los ríos Arauca, Tibú, Catatumbo, Tarra y las quebradas, El Loro, El Carmen, La Medrosa, La Pérdida y Caño Victoria (La Opinión, 2019). Los derrames de hidrocarburos pueden ocasionar daños ambientales y afectar a la población por contaminación de sistemas de abastecimiento de agua, de recursos naturales que son fuente de subsistencia, deterioro de zonas productivas y cultivos, e incluso causar daños a las personas por incendios. Las consecuencias de los derrames se convierten en desastres cuando las alteraciones de las condiciones de las personas, de los bienes y servicios o del medio ambiente son graves (Corponariño, 2017).

El objetivo de este trabajo es proporcionar una idea general sobre como un grupo armado al margen de la ley, por medio de atentados terroristas como la voladura de oleoductos, se convierte en un acción determinante de las afectaciones al medio ambiente, que se transforman en desastre ambiental por la magnitud de la misma operación la cual desencadena en procesos que inducen daños físicos y muchas veces pérdidas de vidas humanas, alterando la existencia de comunidades que habitan en los territorios afectados.
1. Grupo Productos Verdes (GPV), Programa Ingeniería Ambiental, Pamplona, Colombia.

*Autor de correspondencia: javier.vera@unipamplona. edu.co

Identificador:

http://revistareder.com/ handle-0719-8477-2021-072

RECIBIDO

2 de julio de 2020

PUBLICADO

1 de enero de 2021

Formato cita

Recomendada (APA):

Vera Solano, J.A. (2021)

Terrorismo como Detonante

de Desastres: Atentados

Terroristas Contra

Oleoducto Caño Limón

Coveñas en Colombia.

Revista de Estudios

Latinoamericanos sobre

Reducción del Riesgo de

Desastres REDER, 5(1), 126-

136. http://revistareder.com/ handle-0719-8477-2021-072

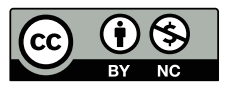

Todos los artículos publicados en REDER siguen una política de Acceso Abierto y se respaldan en una Licencia CreativeCommons Atribución-NoComercial 4.0 Internacional.

Revista de Estudios Latinoamericanos sobre Reducción del Riesgo de Desastres (REDER)

Diseño: Lupe Bezzina 
Para iniciar, se abordan algunos aspectos básicos como la contaminación por hidrocarburos, el análisis del concepto de 'atentado terrorista' y su ocurrencia en Colombia, así como los atentados a la infraestructura petrolera para el caso del oleoducto Caño-Limón Coveñas. Seguidamente, se analiza como estas acciones al margen de la ley ocasionan daños a los componentes del medio ambiente, y por último se hace referencia al desastre ambiental debido a los derrames de hidrocarburos y los desafíos a los que se debe enfrentar el país en la reducción del riesgo por esta clase de eventos.

\section{LOS HIDROCARBUROS Y LA CONTAMINACIÓN}

Los hidrocarburos son compuestos químico-orgánicos que resultan de la combinación del carbono (C) con el hidrógeno $(\mathrm{H})$, abarcan los estados: gaseosos, líquidos, semisólidos y sólidos, como aparecen en la superficie terrestre; o gaseosos y líquidos en las formaciones geológicas en el subsuelo. El origen del hidrocarburo radica en la existencia de una cuenca sedimentaria donde exista la posibilidad de acumulación de sedimentos. La roca madre en una cuenca lodosa es la responsable de la generación del hidrocarburo. Una vez formado migra por el subsuelo a través de rocas permeables y porosas, así como también por la acción de factores estructurales (fallas, diaclasas, pliegues, etc.), hasta conseguir en su trayecto rocas impermeables o arreglos estructurales que permitan el entrampamiento de este. De esta manera se constituye tanto la roca almacén, donde se acumulan los hidrocarburos que se extraen comercialmente (también conocida como yacimiento petrolífero) y la roca sello, secuencia litológica de porosidad y permeabilidad reducidas, la cual sirve como sello a la migración del hidrocarburo y soporta la constitución de una trampa petrolífera (Vílches Fernández \& Ulloa Carcasés, 2015)

En este orden de ideas si determinamos que la contaminación es la introducción de sustancias a cualquiera de los componentes del medio ambiente, agua, aire, suelo, y que puede producir una alteración significativa en el mismo, la contaminación por hidrocarburos constituye un serio riesgo para el medio ambiente, ya que son sustancias altamente persistentes que presentan elevados niveles de bioacumulación y alcanzan altas cotas de toxicidad (Mancera Rodríguez \& Alvarez León, 2005). Hecha la observación anterior se puede inferir que la contaminación por hidrocarburos se puede generar por la manipulación inadecuada de los mismos, el transporte, su comercialización, producción, distribución, los escapes por tuberías, desechos, derrames accidentales o provocados, entre otros. La contaminación por hidrocarburos se presenta cuando estos entran en contacto directo con el suelo, el agua, las plantas y los animales, en general con todo lo que esta sustancia química pueda tocar. Es evidente entonces que, con cada atentado terrorista perpetrado a la infraestructura petrolera, se está generando un nivel de contaminación de altas proporciones que terminan en desastres ambientales siendo estas acciones en la mayoría de los casos, un acto político que tiene como propósito causar daños dramáticos y mortales sobre civiles mientras que crea una atmosfera de miedo e incertidumbre entre la población (Rodríguez Morales, 2012). En otras palabras, lo que se genera por parte de estos actos 0 atentados terroristas es un 'desastre' para muchas de las personas o comunidades afectadas.

\section{TERRORISMO Y ATENTADO TERRORISTA}

Aunque no existe completo consenso sobre el significado de la palabra 'terrorismo' en español, podemos encontrar varios significados que nos pueden dar ciertas luces sobre el tema:

Forma violenta de lucha política, mediante la cual se persigue la destrucción del orden establecido o la creación de un clima de terror e inseguridad susceptible de intimidar a los adversarios 0 a la población en general.

(García Parra, 2014).

Es la dominación por medio del terror, el control que se busca a partir de actos violentos cuyo fin es infundir miedo.

(Mateos Ochoa, 2017).

Ataque $u$ ofensa contra algo que se considera bueno o justo (atentado) con base a los anteriores conceptos es oportuno hablar de atentado terrorista.

(Lobo Fernández, 2014). 
Cuando se utiliza el término 'atentado', se hace referencia a aquellos fenómenos que son generados con una alta violencia y que en la totalidad de los casos son planeados con antelación con el fin de llamar la atención y demostrar cierto tipo de postura ante determinado fenómeno, es, en otras palabras, una agresión o desacato grave a la autoridad u ofensa a un principio $u$ orden que se considera recto (Real Academia Española, 2018).

Un atentado puede ser provocado por un individuo de manera aislada o por una organización terrorista, en cuyo caso la repercusión puede tener consecuencias políticas además de sociales y ambientales. Entonces, un atentado es un acto criminal contra una nación, una autoridad, o contra cualquier bien material o persona, con la clara misión de alterar el orden vigente.

La palabra inglesa attack puede traducirse al español como 'ataque', aunque según el contexto, puede traducirse como 'atentado', 'asalto' o 'agresión'. Para nuestro caso, lo que en inglés sería un terrorist attack será entendido aquí como 'atentado terrorista'.

Según el Diccionario de la Real Academia Española de la Lengua, 'ataque' es la acción de atacar, acometer o emprender una ofensiva, es decir, que tiene un significado mucho más genérico que 'atentado', el cual significa, según el citado diccionario, una agresión contra la vida o la integridad física o moral de alguien. Sobre la base de las consideraciones anteriores podríamos entonces reconocer que las acciones que el Ejército de Liberación Nacional (ELN) realiza en Colombia en contra de la infraestructura petrolera son 'atentados terroristas', que buscan, más allá de llamar la atención, infundir terror en la población y los gobiernos territoriales, alterar el orden social y, como consecuencia última, destruyen el componente ambiental que se encuentra en la zona adyacente a donde se produce el atentado.

Resulta oportuno mencionar que en Colombia existen al menos tres grupos armados al margen de la ley, denominados 'guerrillas', 'paramilitares' y las 'bacrim' (bandas criminales). Entre ellos, la principal fuente que contribuye a generar desastres ambientales en el país es la guerrilla del ELN. Ésta justifica sus ataques con el argumento de que las empresas multinacionales petroleras y mineras se apoderaron de los recursos naturales del país. Contradictoriamente, esta guerrilla es la que más desastres ambientales ha producido en el territorio colombiano.

\section{LOS ATENTADOS TERRORISTAS EN COLOMBIA Y LOS GRUPOS ARMADOS QUE LOS GENERAN}

En el marco de las observaciones anteriores, la guerrilla del Ejército de Liberación Nacional (ELN), surgida en 1964, ataca por primera vez el oleoducto Caño Limón Coveñas en los años 80 (Torrado, 2018). En la siguiente Figura 1, se ofrece una secuencia que representa el proceso de producción de un desastre ambiental detonado por los atentados terroristas antes mencionados. Generado por el grupo terrorista que representa la amenaza constante de atentar contra la infraestructura petrolera, la ocurrencia de la amenaza, es decir el atentado en sí mismo, produce un impacto ambiental que muchas veces el Estado o las comunidades no tienen las capacidades de respuesta, constituyéndose el 'desastre ambiental'.

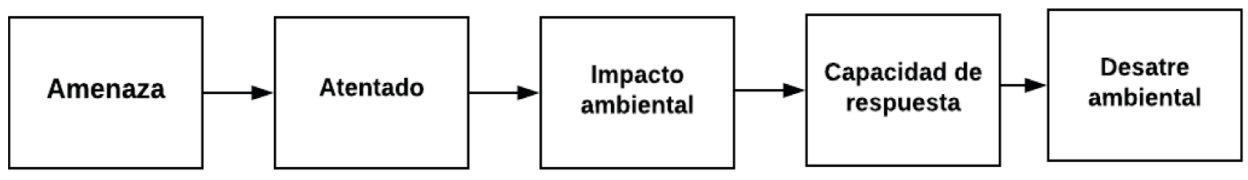

Figura 1. Secuencia de elementos que generan el desastre ambiental Fuente: Autor, 2021

Hablamos de la secuencia del desastre de forma general, ya que esta guerrilla no ha suspendido en forma indefinida sus actuaciones 0 atentados. En relación a esto último podemos mencionar que hasta noviembre del año 1998 en Colombia se presentaron 920 atentados contra la infraestructura petrolera, 575 de ellos en el oleoducto Caño Limón Coveñas, que mediante roturas y abolladuras han perjudicado no solo a los ecosistemas y fuentes de producción y abastecimiento de las comunidades aledañas al oleoducto, sino a regiones por donde este transita (López de Mesa et al., 2006). 


\section{OLEODUCTO CAÑO LIMÓN COVEÑAS, PRINCIPAL OBJETIVO DE ATENTADOS EN COLOMBIA}

El oleoducto Caño Limón Coveñas, que es propiedad de Cenit y es operado por la Vicepresidencia de Transporte y Logística de Ecopetrol (Empresa Colombiana de Petróleos), tiene una extensión de 773,94 kilómetros, y atraviesa 33 municipios, 253 veredas en los departamentos de Arauca, Boyacá, Norte de Santander, Cesar, Magdalena, Bolívar y Sucre. Al año 2015 y en sus 30 años de existencia, este sistema ha sido objeto de 1.293 actos ilícitos como atentados y la instalación de válvulas para el hurto del crudo por parte de grupos al margen de la ley (Ecopetrol, 2015). El oleoducto se construyó en el año 1986, y dos años después ya se tenían reportes de derrame, desde Colombia al territorio venezolano, de cerca de 161,485 barriles de petróleo a través de los ríos Tarra y Catatumbo (Rivas et al., 2009), dejando múltiples daños ambientales (Miranda $\&$ Restrepo, 2005). Desde entonces se han registrado cerca de 1.400 voladuras al ducto a su paso por municipios de Norte de Santander, específicamente por la región del Catatumbo que comprende los municipios de Convención, El Carmen, Teorama, San Calixto, El Tarra, Tibú, Hacarí, Sardinata y La Playa. Además, en el departamento funcionan tres pozos: Campo Dos, Tibú y Río de Oro (Guerrero Useda, 2018), que son fuente de amenaza por el potencial efecto negativo que su afectación, producto de atentados terroristas.

Es evidente entonces que existe una lista extensa de atentados (ver un resumen en Tabla 1) realizados en contra de infraestructura petrolera, los cuales cada vez que se realizan vierten a suelo cientos de barriles de crudo. Solo para el año 2018, la infraestructura petrolera sufrió 107 atentados de los cuales 89 afectaron al oleoducto Caño Limón Coveñas (Agencia EFE, 2019). Para el año 2019, al mes de junio, se han contabilizado aproximadamente 19 ataques a esta infraestructura, generando aproximadamente un derrame de 20 mil barriles de petróleo.

\begin{tabular}{lcl}
\multicolumn{1}{c}{ Fecha o Año } & Barriles derramados & Sector \\
\hline 14 julio de 1986 & 45.743 & $\begin{array}{l}\text { La Donjuana, Municipio del Carmen de Tonchala Norte de } \\
\text { Santander }\end{array}$ \\
\hline Febrero 1988 & 93.952 & Llego al lago Maracaibo Venezuela \\
\hline 15 mayo de 1990 & 14.000 & Zapatosa Cesar \\
\hline Julio 1992 & 45.000 & San Roque, en los ríos Ité y Cimitarra. \\
\hline 2005 & 22.458 & $\begin{array}{l}\text { Ciénagas de Juan Manuel, Aguas Blancas y Aguas negras, } \\
\text { en el estado Zulia, Venezuela }\end{array}$ \\
\hline 25 marzo de 2014 & - & La China, municipio de Toledo, en Norte de Santander, \\
\hline Abril de 2019 & - & $\begin{array}{l}\text { Río Tibucito y Pozo Azul, un sitio turístico de Norte de } \\
\text { Santander. }\end{array}$ \\
\hline
\end{tabular}

Tabla 1. Derrames de crudo producidos los últimos años por atentados terroristas Fuente: Autor, 2021, basado en Revista e+ (2015).

Considerando que los atentados a la infraestructura de oleoducto Caño Limón Coveñas han venido en aumento, podríamos afirmar que también esto conlleva un aumento las afectaciones que este tipo de acciones generan en el medio ambiente y las comunidades. Es evidente entonces que ante la presencia de eventos como los atentados terroristas se hace necesario examinar como los mismos pueden afectar no solo a los recursos naturales sino también al patrimonio, y sobre todo a la población que en ultima instancia termina siendo la más afectada.

\section{IMPACTOS AL MEDIO AMBIENTE POR LOS DERRAMES PRODUCTO DE ATENTADOS TERRORISTAS}

Existen algunos factores que pueden influir en la naturaleza y duración de un derrame, entre ellos podemos encontrar el tipo y cantidad de hidrocarburo derramado (ver Figura 2), el sitio 0 ubicación en donde se presenta el derrame, la velocidad como el contaminante se disipa o diluye por procesos naturales, las técnicas de limpieza con las que se desea controlar el derrame y la sensibilidad de las especies ante el tipo de contaminación (Itopf Ltd., 2011).

Los impactos que los derrames han ocasionado pueden clasificarse básicamente en tres: Humanos, al Patrimonio, y Ecológicos (Cavazos Arroyo et al., 2014). A continuación, se mencionan:

Humanos. Los derrames de crudo también tienen impactos sobre la población, ya que el agua de consumo humano -que de hecho se suministra a la población sin ningún tipo de tratamiento-se 
abastece de acueductos cuyas fuentes son ríos, ocasionalmente contaminados por causa de los derrames. Estos derrames también han dejado sin agua innumerables veces a las comunidades de las zonas aledañas a los ríos, sumándose a ello las consecuencias sobre la salud. (Fundación Ideas Para la Paz, 2015a).

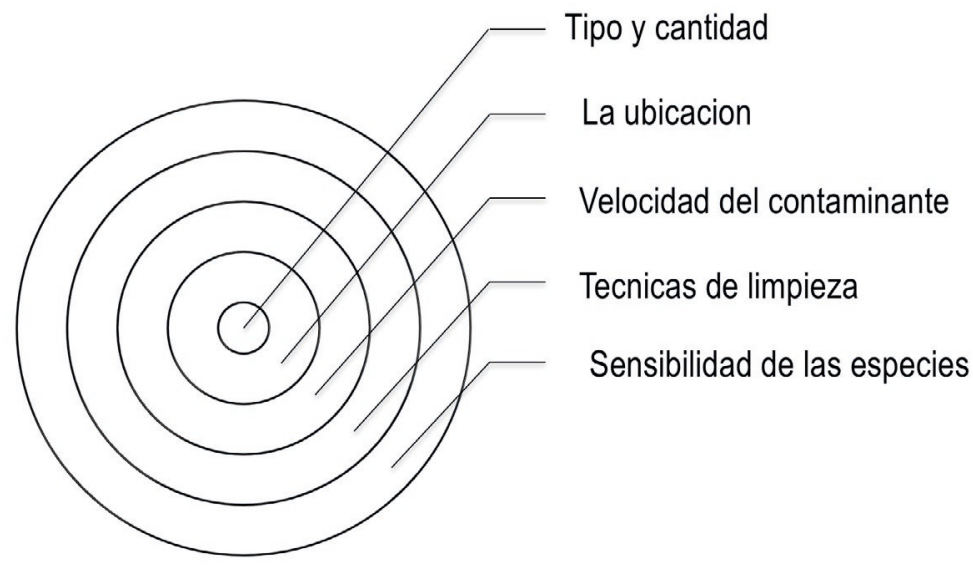

Figura 2. Factores que pueden influir en la naturaleza y duración de un derrame de petróleo Fuente: Autor, 2021, basado en Revista e ${ }^{+}$(2015).

Patrimonio. En el año 2018, el costo de las reparaciones derivadas de los atentados terroristas y de las válvulas ilícitas en los sistemas de transporte, fue cercano a los $\$ 157.000$ millones de dólares americanos (US\$). En ese mismo sentido, los actores armados ilegales han desarrollado sofisticados sistemas para captar recursos petroleros mediante la influencia de partidos políticos, gobiernos locales, organizaciones comunitarias y el uso de la coacción selectiva de la producción donde se extraen los hidrocarburos. Con esto, se genera una estructura de oportunidad para que los grupos armados ilegales se apropien de los recursos públicos. En este contexto, las regalías configuran un botín de fácil acceso para los actores ilegales, quienes en ocasiones se conforman como una institucionalidad paralela o informal (Fundación Ideas Para la Paz, 2015b).

Ecológicos. La afectación de los ecosistemas por los derrames de crudo, producto de los atentados terroristas, generan daños importantes a los hábitats de poblaciones animales terrestres y acuáticas. Los principales impactos causados en el medio por un derrame de hidrocarburos son los siguientes: Bioacumulación; Biomagnificación; Volatilización de hidrocarburos; Olores ofensivos; Contaminación de sedimentos; Contaminación de suelos; Productividad primaria; Adhesión de hidrocarburos a neumatóforos y raíces de mangle; Corrientes; Evaporación; y Hidrocarburos disueltos y dispersos, o HDD, entre otros (Jiménez, 2006).

Son incontables los impactos que se generan por las acciones de los grupos armados ilegales. Según Semana (2019), hay una contradicción en la forma como actúan estos grupos, por un lado, defienden la soberanía de los recursos naturales, pero por otro, dañan ecosistemas y fuentes hídricas. En cuanto a esto, se debe recalcar que la magnitud del derrame suele ser proporcional a los impactos ambientales, por sus implicaciones en la contaminación de cuerpos de agua, muerte de especies y un cambio significativo en los componentes del medio ambiente.

\section{EL DESASTRE DEBIDO A LOS DERRAMES GENERADOS POR LAS ACCIONES TERRORISTAS}

Los criterios de evaluación de los desastres ambientales se suelen centrar en el número de muertes humanas, pero desde una perspectiva ecológica se habla de desastre ambiental independientemente de sus efectos sobre el ser humano, aunque ese desastre pueda ser causado 0 agravado por acciones y políticas humanas (Aledo, s.f). El desastre ambiental es una pérdida para el medio ambiente y a cada uno de sus componentes. Es una perturbación o una alteración, en nuestro caso, a los recursos naturales. Para entender mejor las implicaciones que un derrame de crudo ocasiona al medio debido a las acciones terroristas, es necesario revisar cada uno de los componentes afectados, sea el suelo con la alteración de las condiciones del terreno (Serrano Guzmán et al., 2013), el agua por la película de crudo que impide el transcurso normal del proceso de la fotosíntesis en las plantas acuáticas, y así como en la población del área de influencia del oleoducto, ya que la tendencia de este tipo de desastres acontece principalmente en las zonas 
rurales, teniendo mayor efecto en las poblaciones dedicadas a la agricultura (Mauricio Gutiérrez et al., 2014)

Suelo. En la actualidad los suelos contaminados con hidrocarburos representan el $70 \%$ del total de los ecosistemas impactados (Ortiz et al., 2005). Los derivados de hidrocarburos -gasolina, queroseno, aceites, combustibles, parafinas y asfalto entre muchos otros- no solo impactan la capa superficial del suelo, también existe la posibilidad de ser movilizados hasta aguas subterráneas generando así contaminación o incluso pueden ser transportados por escorrentía, incrementado aún más el daño ambiental (Velásquez Arias, 2017). Durante la producción o la distribución del petróleo y sus derivados, bien sea originados de manera involuntaria o forzosa, liberan una cantidad importante de contaminantes orgánicos con alto contenido de compuestos polares (Serrano et al., 2009), los cuales intervienen negativamente en el suelo. Este hecho se complica porque la mayoría del transporte de los subproductos se hace enterrando las tuberías, consiguiendo adicionalmente contaminar los depósitos de agua subterránea, este tipo de contaminación llega a generar perturbaciones en los suelos y más cuando este tipo de ductos se ven atacados por voladuras por parte de grupos terroristas.

Agua. El impacto que causan los hidrocarburos de forma inmediata es que crea una película sobre la superficie del agua que impide la entrada de la luz en la misma. Es evidente entonces que, dentro del ecosistema fluvial, existan diferentes especies, como es el caso de las algas, que necesitan la llegada de la luz para poder realizar la fotosíntesis. Si esto no ocurre, muchas de ellas pueden morir, afectando toda la cadena alimenticia (Plitt, 2010).

Aire. Una de las consecuencias de los derrames son las emisiones producto de la combustión, el óxido nitroso se produce directamente a partir de la quema de combustible.

Hay dos preocupaciones en relación con la incineración in situ de los derrames de crudo: la producción de una gran masa de humo; y los residuos de material que persisten en el medio una vez que ha cesado la incineración.

El primero puede generar afectaciones al aparato respiratorio, tanto de pobladores como animales, así como afectación a las plantas, ya que el humo conlleva consigo trazas de material particulado que se asienta en las hojas de plantas e impide el proceso normal de la fotosíntesis. El segundo, los residuos que quedan en el suelo, pueden ir cambiando totalmente las características fisicoquímicas del mismo y muchas veces esterilizándolo totalmente.

La combustión del petróleo produce una densa nube de humo de color negro debido a las pequeñas partículas de carbono causadas por la baja eficiencia en la combustión de tales productos. Estas partículas pueden causar importantes problemas respiratorios o agravar los síntomas de pacientes con enfermedades cardiacas o pulmonares. Otras sustancias emitidas a la atmósfera en estas ocasiones son vapor de agua y gases invisibles, como dióxido de carbono, monóxido de carbono, dióxido de azufre y óxidos de nitrógeno. Se trata, en algunas de ellas de gases de efectos invernadero, otras sustancias como los hidrocarburos poliaromáticos (HPA) y los compuestos volátiles orgánicos (CVO) caen a niveles base a escasa distancia del lugar de la incineración (España, s.f)

Fauna. Las aves, por ejemplo, ven sus plumas impregnadas, perdiendo la capacidad de aislamiento que les da el aceite natural de las mismas y terminan muriendo de hipotermia. Otras veces mueren cuando intentan limpiarse de la contaminación, porque ingieren en el proceso los contaminantes del crudo. En este mismo orden y dirección, si un alga tiene sólo un punto de contaminación, los herbívoros que se alimentan de ellas y comen diez algas tendrán 10 puntos de contaminación y los carnívoros que se alimentan de estos herbívoros tendrán miles de puntos de contaminación. Debido a lo anterior, el impacto en los ecosistemas fluviales tiene una huella negativa en los servicios que los humanos reciben de ellos, como agua, alimentos, tierras de cultivo y sitios de recreación, entre otros. Varios estudios también han demostrado que la interacción de los hidrocarburos con estos entornos puede facilitar su entrada en el ciclo de los nutrientes y los diferentes vínculos en la cadena alimentaria, afectando muchas especies como reptiles, anfibios, mamíferos (ver Figura 3) los cuales finalmente pueden ser consumidos por otras especies o por los propios seres humanos (Miranda \& Restrepo, 2005). En efecto también hay que precisar que cuando el atentado al oleoducto produce un derrame que llega a un cuerpo de agua superficial, la muerte de peces es una tragedia, o directamente 'desastre', y más si se da el caso que en ese sitio pudiesen desovar los animales acuáticos, fuente de alimento de comunidades humanas. 


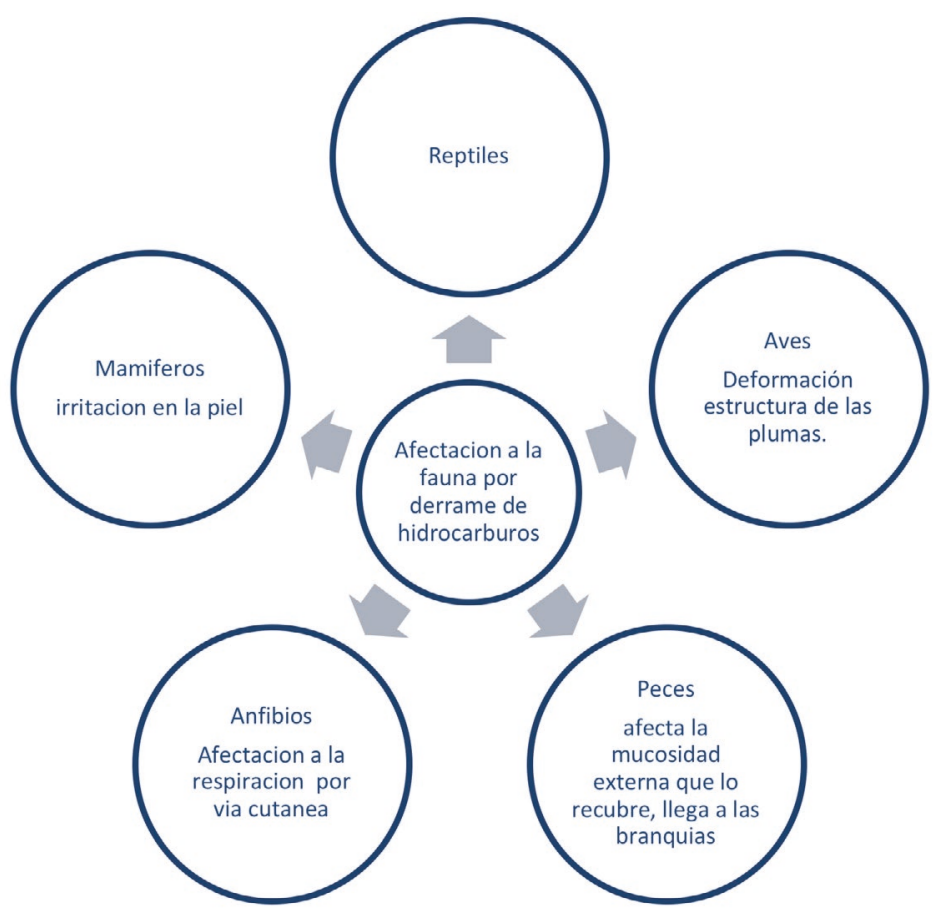

Figura 3. Afectación de la fauna por derrame de hidrocarburos Fuente: Autor, 2021.

Flora. Un desastre ambiental como la devastación acelerada de los bosques, trae consigo graves secuelas como la disminución en la productividad de los suelos. Además, un derrame de crudo conlleva a que las plantas sufran fenómenos de clorosis, necrosis, efectos negativos sobre la reproducción y propagación de estas, inclusive su muerte. El contacto directo de las plantas con el crudo puede producir la absorción de contaminantes presentes en el mismo, además se puede generar la quema del follaje y más aún la perdida de las semillas presentes en las flores o frutos de las especies nativas del área donde se presenta el derrame, impidiendo así la reproducción natural de especies que algunas veces se encuentran en vía de extinción.

La siguiente Tabla 2 resume los impactos del derrame de hidrocarburos en los diferentes componentes mencionados.

A pesar de todas las afectaciones ambientales que se han presentado en Colombia por ocurrencia de derrames de crudo por atentados terroristas, en el país en se ha venido trabajando en el tema de prevención y mitigación del riesgo por estos eventos. Se han establecido planes y programas a partir de la creación de leyes y decretos como por ejemplo: el Decreto 2190 de 1995 mediante el cual se ordena la elaboración y desarrollo del Plan Nacional de Contingencia contra derrames de Hidrocarburos, Derivados y Sustancias Nocivas en Aguas Marinas, Fluviales y Lacustres, como instrumento rector para la prevención, mitigación y corrección de los daños que dichos sucesos puedan ocasionar y en ese mismo sentido las instituciones y comunidades vienen preparándose para superar los desafíos que genera la guerra que tienen los grupos armados al margen de la ley en contra el medio ambiente en Colombia.

\section{DESAFÍOS EN LA REDUCCIÓN DEL RIESGO POR ATENTADOS TERRORISTAS}

En el año 2017, Colombia debido al colapso de los diálogos de paz con el Ejército de Liberación Nacional (ELN), ha complicado su situación de seguridad. Como resultado, hay un incremento de $140 \%$ en la frecuencia de los atentados; de noventa y cinco (95) en el año 2017, a doscientos treinta y uno (231) en el año 2018 (Dinero, 2019). Cabe resaltar que Colombia junto con Iraq, India, Somalia y Nigeria fueron los cinco países del mundo con el mayor número de ataques terroristas en 2018.

La prevención y recuperación, es decir, antes y después de dichos eventos, requiere del trabajo de los gobiernos tanto nacionales, departamentales y municipales, y de las entidades conformadas para la atención de estos. Para hacer frente a estas acciones en Colombia se encuentra el Sistema Nacional para la Prevención y Atención de Desastres (SNPAD), quien coordina en conjunto con los 
Comités Regionales para Prevención y Atención de Desastres (CREPAD) en los departamentos y la Dirección General para la Prevención y Atención Desastres (DGPA) y los Comités Locales de Emergencias (CLE). Todos estos organismos, en caso de desastre o emergencia, tienen planes específicos de acción.

\begin{tabular}{|c|c|c|}
\hline Componente & Impactos & Referencias \\
\hline \multirow{2}{*}{ Agua } & $\begin{array}{l}\text { La primera población afectada por un derrame es el plancton, } \\
\text { en segundo lugar, son afectados los macroinvertebrados y } \\
\text { la última población afectada son los Bentos o población de } \\
\text { macroinvertebrados que viven en los fondos de los ríos y ciénagas }\end{array}$ & $\begin{array}{l}\text { (Menezes Bento et } \\
\text { al., 2003) }\end{array}$ \\
\hline & $\begin{array}{l}\text { La existencia de una película en la interfaz agua-aire tiene por } \\
\text { consecuencia la perturbación de los intercambios gaseosos, lo que } \\
\text { provoca una disminución del proceso de autodepuración por la } \\
\text { disminución de la capacidad de Re-oxigenación del medio. }\end{array}$ & (Galan, 1999) \\
\hline \multirow{2}{*}{ Suelo } & $\begin{array}{l}\text { Las disminuciones de pH se deben a las elevadas cantidades } \\
\text { de hidrocarburos, los cuales causan mediante su degradación la } \\
\text { generación de ácidos orgánicos que acidifican el medio }\end{array}$ & $\begin{array}{l}\text { (Beltrán \& Vela, } \\
\text { 2006) }\end{array}$ \\
\hline & $\begin{array}{l}\text { El petróleo en el suelo causa problemas eco toxicológicos, debido } \\
\text { al potencial tóxico, carcinogénico y mutagénico de los hidrocarburos } \\
\text { que lo componen. }\end{array}$ & $\begin{array}{l}\text { (Rivera Cruz et al., } \\
\text { 2009) }\end{array}$ \\
\hline Aire & $\begin{array}{l}\text { El proceso de combustión del crudo puede generar material } \\
\text { particulado, el cual se adhiere a las hojas de las plantas, } \\
\text { impidiendo su normal proceso de fotosíntesis. Se pueden encontrar } \\
\text { compuestos químicos muy fuertes como aromáticos policíclicos muy } \\
\text { tóxicos. }\end{array}$ & \\
\hline \multirow[b]{2}{*}{ Flora } & $\begin{array}{l}\text { Se presentaron daños visibles, como clorosis y necrosis, en zonas } \\
\text { de afectación. }\end{array}$ & $\begin{array}{l}\text { (Lopez Garcia et } \\
\text { al. 2006) }\end{array}$ \\
\hline & $\begin{array}{l}\text { Dichas sustancias tienden a acumularse y a formar una capa } \\
\text { hidrofóbica, induciendo la fragmentación de los agregados, } \\
\text { causando reducción, inhibición de la cobertura vegetal y la } \\
\text { modificación de las poblaciones microbianas del ambiente edáfico. }\end{array}$ & $\begin{array}{l}\text { (Diaz Martinez et } \\
\text { al., 2013) }\end{array}$ \\
\hline \multirow{2}{*}{ Fauna } & $\begin{array}{l}\text { Los peces no sólo son contaminados por hidrocarburos al ingerir } \\
\text { alimento contaminado, también cuando estas sustancias entran en } \\
\text { su organismo a través de las branquias. Igualmente, puede destruir } \\
\text { los huevos o contaminarlos produciendo descendencias deformes. }\end{array}$ & (AUPEC, 1998) \\
\hline & $\begin{array}{l}\text { El crudo afecta las plumas de las aves, desestabiliza su } \\
\text { termorregulación y facilita la muerte por hipotermia. Los anfibios } \\
\text { son muy susceptibles, ya que el petróleo obstaculiza su respiración } \\
\text { cutánea y sus procesos de transpiración }\end{array}$ & $\begin{array}{l}\text { (Paz Cardona, } \\
\text { 2018) }\end{array}$ \\
\hline Salud & $\begin{array}{l}\text { La exposición al petróleo ocasiona problemas de salud } \\
\text { neurológicos, hematológicos y síntomas irritativos en los habitantes. }\end{array}$ & $\begin{array}{l}\text { (O'Callaghan et } \\
\text { al., 2016) }\end{array}$ \\
\hline
\end{tabular}

Tabla 2. Principales alteraciones por derrames de crudo a los componentes del medio ambiente Fuente: Autor, 2021.

Por su parte, la Empresa Colombiana de Petróleos (Ecopetrol) se encarga de reducir el riesgo tecnológico y operacional de los oleoductos utilizando equipos automatizados que permiten alertar y atender oportunamente cualquier situación anormal. Con estas nuevas tecnologías se accede a articular todo el sistema del oleoducto y mantener activo el transporte de crudo a pesar de cualquier eventualidad, tratando así de reducir los riesgos y salvaguardar la integridad de las personas y la afectación al medio ambiente.

Cabe agregar que, ante una emergencia o desastre, es Ecopetrol quien activa el plan de contingencia para atender la situación, y quien notifica a los Consejos Municipales de Gestión del Riesgo y Desastres en el municipio. Es allí donde la comunidad juega un rol muy importante, porque muchas veces es ella la que se enfrenta directamente a la situación tratando de contribuir a la búsqueda de la solución del problema debido a la presencia de estos acontecimientos adversos, en otras palabras, suelen ser los primeros en responder. 


\section{CONCLUSIONES}

Un atentado terrorista es una agresión contra alguien o algo, lo cual puede trasferir tanto daño que conlleva a devastar o destruir. El terrorismo es una de las amenazas más graves para la seguridad, para la paz, para la población y sobre todo para el medio ambiente, cuando se trata de infringir daño a procesos que pueden converger en fuentes de desastres como es el ejemplo de voladuras de oleoductos que conllevan a la transformación total de ecosistemas acuáticos y terrestres.

Si partimos del concepto de desastre como un evento calamitoso, repentino o previsible que trastorna seriamente el funcionamiento de una comunidad o sociedad y causa unas pérdidas humanas, materiales, económicas o ambientales que desbordan la capacidad de la comunidad 0 sociedad afectada para hacer frente a la situación a través de sus propios medios, podemos concluir que los grupos subversivos en Colombia son uno de los principales actores y determinantes en la generación de impactos ambientales, sociales y económicos, los cuales terminan convirtiéndose en desastre.

El vertimiento de miles de barriles de crudo por atentados terroristas, generan la contaminación de capa superficial del suelo, siendo este un impedimento para la presentación del ciclo básico de regeneración del mismo. En este sentido, el fenómeno de la película o capa que se forma luego del derrame es la principal causa de la perturbación de los componentes afectados del medio ambiente, ya que ella impide los intercambios normales de los ecosistemas terrestres y acuáticos.

Los atentados terroristas por parte de los grupos subversivos se han convertido en una amenaza constante contra la integridad social, económica y ambiental en Colombia, convirtiendo esta acción intencionada en un castigo para los ecosistemas. Es necesario que los individuos y organizaciones que hacen parte de estos atentados consideren que sus acciones tienen también impactos ambientales y en última instancia en la vida de las personas en los territorios en conflicto. Atentar contra el medio ambiente, y en especial con el derrame de crudo, es un crimen de acuerdo a la ley y un desastre en general, ya que afecta la integridad física y emocional de las personas y de los demás seres vivos.

\section{REFERENCIAS}

Agencia EFE. (2019). Dos ataques al oleoducto de Caño Limón Coveñas. El Heraldo. Recuperado de: https://www.elheraldo.co/colombia/dos-ataques-oleoducto-de-cano-limon-covenas-620928

Aledo, A. (s.f). La crisis ambiental y su interpretación. UA.

AUPEC. (1998). Ciencia al Día. UNIVALLE. Recuperado de: http://aupec.univalle.edu.co/informes/ marzog8/gorgona.html

Beltrán, P., \& Vela, C. (2006). Suelos contaminados con hidrocarburos y su efecto en la formación de agregados del suelo en la Venta, Tabasco. Universidad Autónoma Metropolitana.

Cavazos Arroyo, J., Perez Armendáriz, B., \& Mauricio Gutierrez, A. (2014). Afectaciones y consecuencias de los derrames de hidrocarburo en suelos agricolas de Acatzingo, Puebla, Mexico. Agricultura, Sociedad y Desarrollo, 11(4), 539-550.

Corponariño. (2017). Guia para la prevencion de derrames de hidrocarburos. INVEMAR.

Diaz Martinez, E., Alarcon, A., Ferrera Cerrato, R., Almaraz Suarez, J., \& Garcia Barradas, O. (2013). Crecimiento de Casuarina equisetifolia (Casuarinaceae) en suelo con diesel, y aplicación de bioestimulación y bioaumentación. Rev. Biol. Trop, 61(3), 1039-1052.

Dinero. (10 de abril de 2019). Colombia, en un deshonroso Top 5. Dinero. Recuperado de: https://www.dinero.com/empresas/confidencias-on-line/articulo/ colombia-de-nuevo-en-top-5-de-terrorismo/269504

Ecopetrol. (11 de diciembre de 2015). Ecopetrol. Recuperado de: https://www.ecopetrol.com.co

España, M. (s.f). Ministerio para la transición Ecológica. MITECO. Recuperado de: https://www.miteco. gob.es/es/costas/temas/proteccion-medio-marino/plan-ribera/contaminacion-marina-accidental/ incineracion.aspx

Fundación Ideas Para la Paz. (2015a). El ELN y la industria petrolera: Ataques a la infraestructura en Arauca: Área Dinámicas del Conflicto y Negociaciones de Paz. Fundación Ideas Para la Paz. Recuperado de: http://cdn.ideaspaz.org/media/website/document/55411b8azccab.pdf 
Fundación Ideas Para la Paz. (2015b). El ELN y la industria petrolera: ataques a la infraestructura en Arauca. Área Dinámicas del Conflicto y negociaciones de Paz. Fundación Ideas Para la Paz. Recuperado de: http://cdn.ideaspaz.org/media/website/document/55411b8azccab.pdf

Galan, P. (1999). Contaminación Petrolera. Signos Universitarios, (35), 179-19o.

García Parra, E. (2014). Terrorismo y turismo. Universitat Jaume I. http://hdl.handle.net/10234/152645

Gobernación del Casanare. (2011). Manual Educativo para la prevención de emergencias y desastres. Gobernación del Casanare

Guerrero Useda, M.E. (2018). Ruptura de oleoductos por interferencia externa, daño ambiental y sostenibilidad en Colombia. Producción + limpia, 13(2), 7-13.

Issa Tejada, L.F. (2015). Efectos del Terrorismo en los Oleoductos de Colombia. Universidad Militar Nueva Granada. Repositorio Universidad Militar. http://hdl.handle.net/10654/7789

Itopf Ltd. (2011). Efectos de la contaminacion por hidrocarburos en el medio marino. Impact PR \& Design Limited.

Jimenez Buitrago, D.L. (2006). Estudio de impacto ambiental generado por un derrame de hidrocarburos sobre una zona estuarina, aledaña al terminal de Ecopetrol en Tumaco. Universidad de La Salle.

La Opinión. (27 de enero de 2019). Ataques a la infraestructura petrolera, la otra cara de la lucha armada del ELN. La Opinión. Recuperado de: https://www.laopinion.com.co/politica/ ataques-la-infraestructura-petrolera-la-otra-cara-de-la-lucha-armada-del-eln-170193\#OP

Lobo Fernández, J. (2014). El terrorismo entre la guerra y la paz: aproximaciones desde la antropología filosófica y el derecho internacional. Estudios Internacionales, 46(178), 9-34. http://doi. org/10.5354/0719-3769.2014.32514

Lopes, L.J., Neto, M.S., Terra Argoud, A.R., de Camargo Junior, J.B., \& Ignacio Pires, S. R. (2015). Uma análise das práticas de green supply chain management. Revista de Administração da UNIMEP, 13(1), 165-188.

Lopéz de Mesa, J., Quintero, G., Guevara Vizcaino, A.L., Jaimes Cáceres, D.C., Gutierrez Riaño, S.M., \& Miranda Garcia, J. (2006). Bioremediación de suelos contaminados con hidrocarburos derivados del petróleo. Nova Publicacion Cientifica, 82-90.

Lopez Garcia, E., Zabala Cruz, J., \& Palma López, D.J. (2006). Characterization of Plant Communities in an Area Affected by Hydrocarbon Spill. Terra Latinoamericana, 24(1), 17-26.

Mancera Rodríguez, N.J., \& Alvarez León, R. (2005). Estado delconocimiento de las concentraciones de hidrocarburos y residuos organoclorados en peces dulceacuícolas de Colombia. Dahlia - Rev. Asoc. Colomb. Ictio, 8, 89-103.

Mateos Ochoa. (2017). Técnicas de protección de personas. SEAD o112. Vigilancia y seguridad privada y de personas. Editorial I.C.

Mauricio Gutiérrez, A., Cavazos Arroyo, J., \& Pérez Armendáriz, B. (2014). Afectaciones y consecuencias de los derrames de hidrocarburos en suelos agrícolas de Acatzingo, Puebla, Mexico. ASyD, 11, 539550. http://ref.scielo.org/37byn4

Menezes Bento, F., De Oliveira Camargo, F.A., Okeke, B., \& Frankenberger, W.T. (2003). Bioremediation of soil contaminated by diesel oil. Braz. J. Microbiol, 34, 65-68.

Miranda, D., \& Restrepo, R. (2005). Los derrames de petróleo en ecosistemas tropicales - Impactos, consecuencias y prevención. La experiencia de Colombia. Actas de la Conferencia Internacional sobre Derrames de Petróleo. International Oil Spill Conference Proceedings, 2005(1), 571-575.

O'Callaghan, G.C., Orta, M.M., \& Kogevinas, M. (2016). Health effects of non-occupational exposure to oil extraction. Environmental Health 12(56), 1-4. https://doi.org/10.1186/s12940-016-0140-1

Ortiz, E., Núñez, R., Fonseca, E., Alamazán, V., Cabranes, Y., Miranda, A., Borges, G., et al. (2005). Biorremediacion de suelos contaminados con hidrocarburos. Revista Contribución a la Educación y la Protección del Medio Ambiente, 6, 51-6o.

Paz Cardona, A. J. (6 de abril de 2018). Salvando la fauna afectada por el derrame de petróleo en Colombia. Mongabay Latam. Recuperado de https://es.mongabay.com/2018/04/ rescate-fauna-derrame-petroleo-colombia/

Plitt, L. (28 de abril de 2010). ¿Cuál es el verdadero impacto de un derrame de petróleo?. BBC Mundo. Recuperado de: https://www.bbc.com/mundo/ internacional/2010/04/100428 derrame petroleo claves lp 
Real Academia Española. (2018). Atentado. Diccionario de la Lengua Española. Recuperado de: $\underline{\text { https:// }}$ dle.rae.es/?id= ${ }_{4} \mathrm{CwaGrn} \mid{ }_{4} \mathrm{CykJbf}$

Rivas, Z., Sánchez, J., Troncone, F., Márquez, R., Ledo de Medina, H., Colina, M. et al. (2009). Nitrógeno y fósforo totales de los ríos tributarios al sistema lago de Maracaibo, Venezuela. Interciencia, 34(5), 308-314.

Rivera Cruz, M., Ferrera Cerrato, R., Sanchez Garcia, P., Volke Haller, V., Fernández Linares, L., \& Rodríguez Vásquez, R. (2009). Contaminación de aguas subterráneas por hidrocarburos líquidos livianos en fase no acuosa. Ciencia Ahora, (22), 20-29.

Rodríguez Morales, T.G. (2012). El terrorismo y nuevas formas de terrorismo. Espacios publicos, 15(33), 72-95. https://www.redalyc.org/pdf/676/67622579005.pdf

Semana. (7 de noviembre de 2019). Voladuras una cruda arma de guerra. Especiales semana sostenible. Recuperado de: http://especiales.sostenibilidad.semana.com/voladuras-de-oleoductos-encolombia/index.html

Serrano, A., Tejada, M., Gallego, M., \& Gonzales, J.L. (2009). Evaluation of Soil Biological Activity after a Diesel Fuel Spill. Science of the Total Environment, 407(13), 4056-4051. https://doi.org/10.1016/j. scitotenv.2009.03.017

Torrado, S. (2018). ¿Cuál es el origen del ELN?. Canal Uno. Recuperado de: https://canalı.com.co/ noticias/origen-del-eln/

Velásquez Arias, J.A. (2017). Contaminación de suelos y aguas por hidrocarburos en Colombia. Análisis de la fitorremediación como estrategia biotecnológica de recuperación. Revista de Investigación Agraria y Ambiental, 8(1), 151-167. https://doi.org/10.22490/21456453.1846

Vílches Fernández, O.E., \& Ulloa Carcasés, M. (2015). Evaluación del impacto ambiental por presencia de hidrocarburos en el fundo Los Clavelitos. Mineria y Geologia, 31(3), 91-10. 